\title{
Quality of life and dyspnoea in patients treated with bosentan for idiopathic pulmonary fibrosis (BUILD-1)
}

\author{
G. Raghu*, T.E. King Jr" J. Behr, K.K. Brown+, R.M. du Bois ${ }^{+}$, I. Leconte ${ }^{\S}$, \\ S. Roux ${ }^{\S}$ and J. Swigris ${ }^{+}$
}

ABSTRACT: No therapy is known to improve health-related quality of life (HRQoL) or dyspnoea in patients with idiopathic pulmonary fibrosis. The present study investigated longitudinal changes in HRQoL and dyspnoea and explored the effects of bosentan on these end-points during the Bosentan Use in Interstitial Lung Disease (BUILD)-1 trial.

In total, 154 subjects received oral bosentan $(n=71)$ or placebo $(n=83)$. Changes in HRQoL and dyspnoea from baseline to month (M) 6 and up to M12 were measured using the St George's Respiratory Questionnaire (SGRQ), 36-item short-form health survey (SF-36), Transition Dyspnoea Index and Borg dyspnoea index.

Overall, minimal changes occurred in measures of HRQoL and dyspnoea among placebotreated subjects during the study. The effects of bosentan treatment on HRQoL and dyspnoea in the all-treated population were minimal. However, in the subset of subjects who had undergone surgical lung biopsy for diagnosis of idiopathic pulmonary fibrosis, treatment effects were observed up to M12 in the impact domain of the SGRQ and the physical functioning, general health and role emotional domains of the SF-36.

HRQoL and dyspnoea changed minimally during the course of the present study. Observations from exploratory analyses suggested benefits of bosentan on HRQoL among patients who had undergone surgical lung biopsy for diagnosis, and they merit further investigation.

KEYWORDS: Bosentan, dyspnoea, health-related quality of life, idiopathic pulmonary fibrosis

I diopathic pulmonary fibrosis (IPF) is a progressive severe disease with a bleak prognosis. Median survival is very poor, estimated to be 3 yrs from diagnosis [1]. The predominant symptom of IPF is dyspnoea, which has a strong influence on health-related quality of life (HRQoL) [2]. The limited data available suggest that no pharmacological agent improves survival, symptoms or HRQoL in patients with IPF. According to a recent hypothesis, IPF is an epithelial-fibroblastic disease [3], and the pathogenesis of IPF results from the convergence of several complex profibrotic pathways, leading to aberrant and dysregulated repair of injured lung tissue. Recent therapeutic trials have targeted molecules within these profibrotic cascades.

Endothelin (ET) 1 is a potent mediator of fibrosis, inflammation, hypertrophy and vasoconstriction, and is implicated in the pathophysiology of lung fibrosis $[4,5]$. The deleterious effects of ET- 1 are mediated via two receptors, $\mathrm{ET}_{\mathrm{A}}$ and $\mathrm{ET}_{\mathrm{B}}$. Recently, the Bosentan Use in Interstitial Lung
Disease (BUILD)-1 trial evaluated the oral dual ET receptor antagonist bosentan for the treatment of IPF [6]. Although bosentan did not show superiority over placebo in the primary end-point of 6-min walking distance (6MWD), a trend favouring bosentan was observed in the combined outcome of time to disease progression or death. This effect was most pronounced in a posthoc analysis of the predefined subset of patients who had undergone surgical lung biopsy (SLB) for diagnosis of IPF.

Like patients with other life-shortening diseases, patients with IPF value the quality of their lives, as well as the duration of their life expectancy. HRQoL is, therefore, an important outcome requiring systematic assessment in therapeutic trials in IPF [7]. The BUILD-1 trial was one of the largest studies to assess the impact of medical therapy on HRQoL and dyspnoea in IPF patients. The aim of the present exploratory analysis was to examine longitudinal changes in HRQoL and dyspnoea in IPF and to explore whether or not
AFFILIATIONS

*University of Washington, Seattle, WA,

\#University of California, San Francisco, CA,

${ }^{+}$National Jewish Medical and Research Center, Denver, CO, USA, "Dept of Internal Medicine I, University of Munich, Munich, Germany, and

${ }^{\S}$ Actelion Pharmaceuticals, Allschwil, Switzerland.

CORRESPONDENCE

G. Raghu

Division of Pulmonary and Critical Care Medicine, Campus Box 356166 University of Washington School of Medicine

Seattle

WA 98195-6522

USA

E-mail: graghu@u.washington.edu

Received:

Dec 122008

Accepted after revision:

Aug 062009

First published online:

Aug 132009

European Respiratory Journal Print ISSN 0903-1936

Online ISSN 1399-3003 
bosentan treatment had beneficial effects on these outcomes. It was hypothesised that bosentan would mitigate impairment of HRQoL and dyspnoea to a greater extent than placebo, particularly among the subset of subjects who had undergone SLB for diagnosis of IPF.

\section{METHODS}

\section{Study subjects}

The inclusion and exclusion criteria and the study protocol for the BUILD-1 trial have been described previously [6]. Briefly, the randomised controlled trial enrolled subjects with a welldefined diagnosis of IPF [8] and excluded subjects who had interstitial lung disease due to conditions other than IPF, very severe restrictive pulmonary physiology, obstructive lung disease, or echocardiographic evidence of severe pulmonary hypertension (systolic pulmonary pressure of $\geqslant 50 \mathrm{mmHg}$ ) or congestive heart failure [6]. All of the subjects provided written informed consent to participation in the study, which was approved by the appropriate independent ethics committees or institutional review boards. The study was conducted in accordance with the principles of the Declaration of Helsinki and local laws and guidelines for good clinical practice.

\section{Study design and assessment tools}

The objectives of the present exploratory analyses were to examine changes over time in HRQoL and dyspnoea, and to evaluate the effects of bosentan on changes from baseline in HRQoL and dyspnoea during the BUILD-1 trial [6]. Eligible subjects were randomised 1:1 to receive $62.5 \mathrm{mg}$ oral bosentan twice daily for 4 weeks, up-titrated to $125 \mathrm{mg}$ twice daily thereafter, or matching placebo. Subjects completed HRQoL and dyspnoea assessments at baseline, month (M) 6, and M12, or sooner if the study medication was discontinued prematurely (up to M12).

\section{Health-related quality of life}

Assessment of HRQoL was performed using two instruments, the St George's Respiratory Questionnaire (SGRQ) [9] and the Medical Outcomes Study 36-item short-form health survey (SF-36) [10]. Questionnaires were provided to subjects in their local language, after review by native speakers and persons knowledgeable in this field, prior to and following backtranslation into English.

The SGRQ is a self-administered respiratory-specific instrument that comprises three domains, symptoms, activity, and impacts. Each SGRQ domain is scored 0-100, with higher scores indicating worse HRQoL [9]. Among patients with chronic obstructive pulmonary disease, a change in the total or any SGRQ domain score of 4 points is considered a minimum important difference [11].

The SF-36 is a self-administered generic instrument that measures health status and well-being. It comprises eight domains, individually scored 0-100. Higher scores correspond to better HRQoL [10]. The minimum important difference for SF-36 domain scores has not been clearly established; however, some investigators consider these to be in the range 3-5 points [12]. The SGRQ and SF-36 were selected for the present trial because each had performed well in prior cross-sectional studies enrolling subjects with IPF [7], providing each instrument with a foundation of validity for use in this population.

\section{Dyspnoea}

Dyspnoea was assessed using two instruments: 1) the Borg dyspnoea scale [13], and 2) the Baseline Dyspnoea Index (BDI) and Transition Dyspnoea Index (TDI) [14]. The Borg scale is a simple self-administered visual analogue scale that scores the extent of dyspnoea from 0 (no dyspnoea) to 10 (most severe dyspnoea). Subjects were asked to gauge dyspnoea using the Borg scale immediately following measurement of the 6MWD.

The BDI was assessed only at baseline, at rest and prior to assessments of 6MWD and pulmonary function. Changes in dyspnoea from baseline to M6 and up to M12 were assessed using the TDI, which was performed at rest and prior to assessments of 6MWD and pulmonary function [14]. The BDI and TDI each comprise three domains, functional impairment, magnitude of task and magnitude of effort. The BDI evaluates the extent of dyspnoea on a scale that ranges from 0 (very severe impairment) to 4 (no impairment) for each of three domains [14]. The BDI total score is the sum of the three domain scores and ranges $0-12$. The TDI score ranges from -3 (major deterioration) to 3 (major improvement) for each domain, with baseline status (BDI score) as the comparator. The TDI total score is the sum of the three domain scores and ranges -9-9. Changes in TDI total score of $\geqslant 1$ were considered clinically meaningful [15].

\section{Statistical methods}

Baseline measurements of HRQoL and dyspnoea, and changes from baseline in these parameters, were summarised as median with 95\% confidence interval (CI) unless stated otherwise. The Wilcoxon two-sample test was used to examine differences between treatment groups in HRQoL and dyspnoea from baseline to M6 and from baseline up to M12. Differences between responses observed in bosentan- versus placebo-treated patients were expressed as placebo-corrected median treatment effects, and were estimated using the twosample Hodges-Lehmann estimator, which estimates the median of the treatment differences.

Comparative analyses were performed in patients who had undergone at least one valid post-baseline assessment (alltreated population), and in a subset of subjects who had undergone SLB for diagnosis (SLB subset). Unless stated otherwise, results are presented for the all-treated population. Only patients with valid baseline values were included in the analysis of each parameter. Data were not replaced for patients with missing baseline values; missing data were handled in accordance with published recommendations [9, 10]. For patients with missing post-baseline scores, analyses were performed with the last observation carried forward. In cases of worsening disease or death that precluded data collection, imputed values of 100 for the SGRQ, 0 for the SF-36, 10 for the Borg dyspnoea index and -9 for the TDI were applied.

\section{RESULTS}

\section{Baseline characteristics and patient disposition}

Of the 158 subjects randomised ( $n=74$ for bosentan; $n=84$ for placebo), 154 received at least one dose of study medication, had at least one valid post-baseline value for the primary end-point 
and were included in the all-treated population $(n=71$ for bosentan; $n=83$ for placebo). Treatment groups were generally well matched with regards to demographics and baseline characteristics [6], including measures of HRQoL (table 1). The mean exposure to study drug was 54.0 and 56.1 weeks in the bosentan and placebo groups, respectively.

\section{Health-related quality of life}

All-treated population

As demonstrated in previous studies and compared with general population norms, considerable impairment of HRQoL was observed at baseline (table 1). Among subjects who received placebo, minimal changes in median measures of HRQoL from baseline to M6 and up to M12 were observed during the course of the study (figs. 1 and 2). At M6, a change from baseline in SGRQ total score indicated improvement in bosentan-treated patients; however, up to M12, no differences were observed between treatment groups in any domain of the SGRQ (data not shown).

When quality of life was assessed using the SF-36, no differences were observed between treatment groups in any domain at M6. Up to M12, a change from baseline in the role emotional domain of placebo-treated patients suggested improvement in bosentan-treated patients (data not shown).

\section{SLB subset}

From baseline to M6, improvements were observed in the impacts domain and total score of the SGRQ among patients who had undergone SLB for diagnosis and received bosentan treatment (data not shown). Changes in SGRQ score from baseline up to M12 are illustrated in figure 3a.

There were no differences between treatment groups in change in SF-36 scores at M6 (data not shown). Changes in SF-36 scores from baseline up to M12 are illustrated in figure 3b.

\section{Dyspnoea}

\section{All-treated population}

Minimal changes were observed during the course of the study among placebo-treated subjects who completed assessments of dyspnoea at baseline, M6 and up to M12. The median (95\% CI) Borg scores for these patients were $2.0(2.0-3.0)$ at baseline, 3.0 (2.0-3.0) at M6 and 3.0 (3.0-3.0) up to M12 $(n=83)$. Median changes in dyspnoea measured using the TDI at M6 and up to M12 were $-1.0(-2.0-0.0)$ and $-3.0(-3.0-0.0)$, respectively $(n=82)$.

No differences between treatment groups were observed in Borg scores either at baseline or in changes from baseline at M6 or up to M12 (data not shown). At M6, TDI scores declined from baseline in the placebo group but not in the bosentan group (data not shown). The treatment effect of bosentan at M6 was an increase in TDI of 1.1 (95\% CI 0.0-2.8; $\mathrm{p}=0.02)$. Up to M12, the median TDI declined in both groups (data not shown). At M6, the number of subjects with improved dyspnoea identified by a TDI of $\geqslant 1$, the reported minimum important difference for this instrument, was $18(26.9 \%)$ in the bosentan group and $10(12.2 \%)$ in the placebo group, corresponding to a relative risk of $2.2(95 \%$ CI 1.1-4.5). This effect was not apparent up to M12 (relative risk 1.0; 95\% CI 0.5-2.0).

\begin{tabular}{|c|c|c|c|}
\hline \multirow[t]{2}{*}{ TABLE 1} & \multicolumn{3}{|c|}{$\begin{array}{l}\text { Baseline health-related quality of life in the all- } \\
\text { treated population, assessed using two } \\
\text { instruments }\end{array}$} \\
\hline & & Bosentan & Placebo \\
\hline \multicolumn{4}{|l|}{ SGRQ } \\
\hline \multicolumn{2}{|c|}{ Symptoms components } & $51.2(44.7-56.0)^{\bullet}$ & $54.8(47.9-59.1)^{+}$ \\
\hline \multicolumn{2}{|c|}{ Activity components } & $59.5(53.6-66.1)^{\S}$ & $59.5(56.0-65.6)^{f}$ \\
\hline \multicolumn{2}{|c|}{ Impacts components } & $32.9(25.0-40.3)^{\S}$ & $30.5(25.7-38.4)^{\# \#}$ \\
\hline \multicolumn{2}{|c|}{ Total score } & $43.9(36.6-52.1)^{\pi}$ & $44.3(40.2-50.0)^{++}$ \\
\hline \multicolumn{4}{|l|}{ SF-36 } \\
\hline \multicolumn{2}{|c|}{ Physical functioning } & $55.0(40.0-65.0)^{\bullet}$ & $47.5(35.0-55.0)^{+}$ \\
\hline \multicolumn{2}{|c|}{ Role physical } & $25.0(25.0-50.0)^{5}$ & $25.0(25.0-50.0)^{\# \#}$ \\
\hline \multicolumn{2}{|c|}{ Bodily pain } & $74.0(64.0-100.0)^{\circ}$ & $64.0(61.0-74.0)^{+}$ \\
\hline \multicolumn{2}{|c|}{ Social functioning } & $75.0(75.0-87.5)^{\bullet}$ & $81.3(62.5-87.5)^{+}$ \\
\hline \multicolumn{2}{|c|}{ Mental health } & $80.0(68.0-84.0)^{5}$ & $76.0(68.0-80.0)^{+}$ \\
\hline \multicolumn{2}{|c|}{ Role emotional } & $100.0(66.7-100.0)^{\circ}$ & $100.0(66.7-100.0)^{\# \#}$ \\
\hline \multicolumn{2}{|c|}{ Vitality } & $50.0(40.0-55.0)^{5}$ & $50.0(40.0-55.0)^{+}$ \\
\hline \multicolumn{2}{|c|}{ General health } & $47.0(37.0-57.0)^{\circ}$ & $46.0(40.0-52.0)^{+}$ \\
\hline \multicolumn{4}{|c|}{ 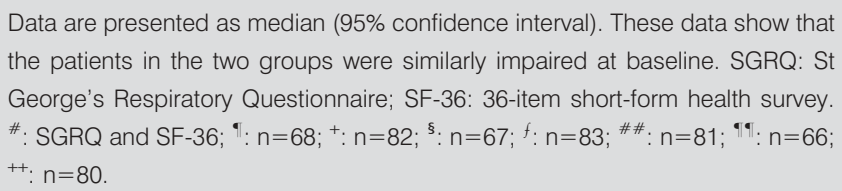 } \\
\hline
\end{tabular}

\section{SLB subset}

There were no differences in baseline Borg scores between treatment groups, and, at M6, the median change from baseline in Borg score was 0.0 (95\% CI 0.0-0.0) in each group. Up to $\mathrm{M} 12$, the Borg score was unchanged in the bosentan group and had changed to $1.0(0.0-1.0)$ in the placebo group, yielding a treatment effect of bosentan of -0.7 (95\% CI -1.4-0.0; $\mathrm{p}=0.03$ ).

A treatment effect of bosentan of $1.3(95 \%$ CI $0.0-2.9 ; \mathrm{p}=0.01)$ was observed in the TDI at M6. Up to M12, the median TDI

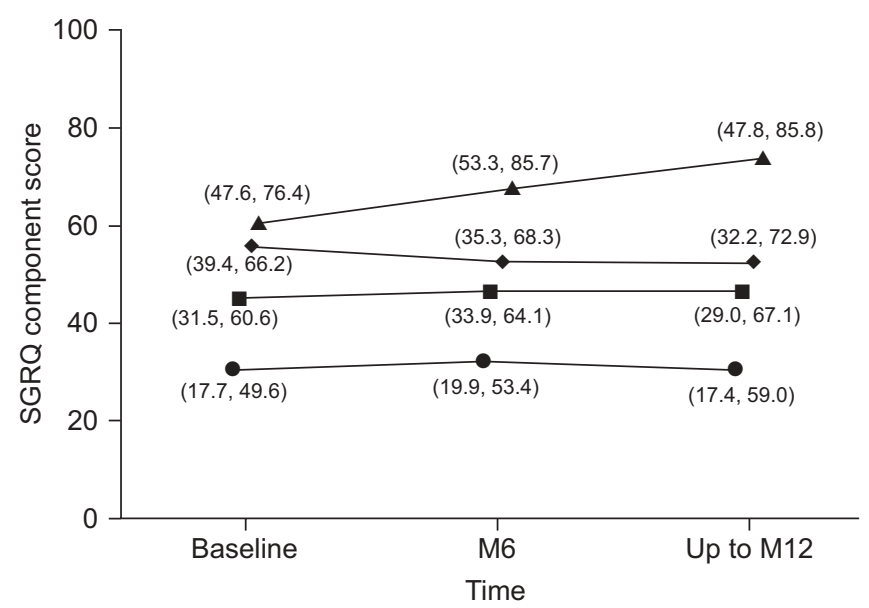

FIGURE 1. Changes in St George's Respiratory Questionnaire (SGRQ) component scores ( : symptoms; $\mathbf{\Lambda}$ : activity; $\bullet$ : impacts; $\mathbf{\square}$ : total) in placebotreated patients in the all-treated population. Lower scores indicate improved health-related quality of life. Data are presented as medians, with interquartile ranges in parentheses. $\mathrm{M}$ : month. 


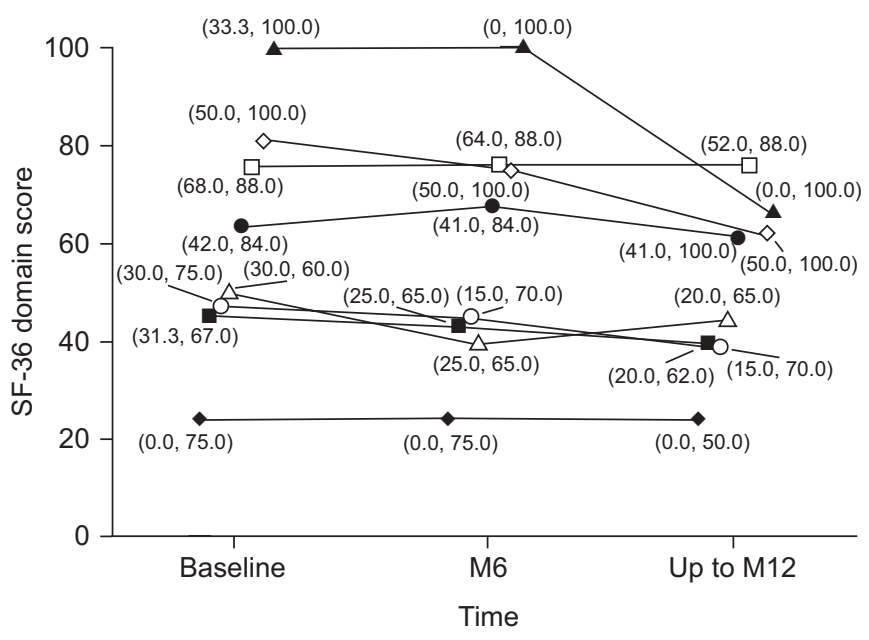

FIGURE 2. Changes in 36-item short-form health survey (SF-36) domain scores $(\triangle$ : vitality; $\boldsymbol{\Delta}$ : role emotional; $\bigcirc$ : physical functioning; $\bullet$ : pain index; $\diamond$ : social functioning; $\diamond$ : role physical; $\square$ : mental health index; $\mathbf{\square}$ : general health) in placebo-treated patients in the all-treated population. Higher scores indicate improved health-related quality of life. Data are presented as medians, with interquartile ranges in parentheses. M: month.

declined in both treatment groups (data not shown). At M6, the number of subjects in the SLB subset with improved dyspnoea identified by a TDI of $\geqslant 1$ was $12(25.0 \%)$ in the bosentan group and four $(8.2 \%)$ in the placebo group, corresponding to a relative risk of 3.1 (95\% CI 1.1-8.8). This effect was not apparent up to M12 (relative risk 0.9; 95\% CI 0.4-2.1).

\section{Safety}

The safety profile of bosentan in the BUILD- 1 trial has been reported previously [6]. The observed incidences of pulmonary adverse events in the safety population were generally less frequent in the bosentan group versus the placebo group, including cough (17.6 versus $27.4 \%$ ), worsening of IPF (16.2 versus $23.8 \%$ ) and exacerbation of dyspnoea (13.5 versus $19.0 \%$ ). The observed incidence of nasopharyngitis and events related to increased liver aminotransferase levels in bosentan-treated patients (13.5 and $13.5 \%$, respectively) were greater than those observed in placebo-treated patients (4.8 and 1.2\%, respectively).

\section{Data and instrument issues}

Domains from both the SGRQ and SF-36 exhibited floor and ceiling effects, i.e. a proportion of subjects in the all-treated population exhibited domain scores at either the lowest or highest possible values for these instruments. The proportion of patients who exhibited floor and ceiling effects in the SGRQ was $\leqslant 3.0 \%$ for all domains except activity, in which $10.6 \%$ of bosentan-treated patients and $11.3 \%$ of placebo-treated patients exhibited the worst possible scores. The proportion of patients who exhibited floor and ceiling effects in the SF-36 was consistently low for the domains of physical functioning, general health, vitality and mental health. In the remaining domains, $18.8-58.2 \%$ of patients in each treatment group exhibited the best possible scores. In the role physical and role emotional domains of the SF-36, 20.9-46.3\% of patients in each treatment group exhibited the worst possible scores.

Within the proportion of patients who dropped out of the study, baseline HRQoL scores were similar in each treatment arm (data not shown). A number of subjects had at least one missing post-baseline value for any domain of any instrument at M6 and up to M12 (table 2). For these patients, analyses were performed with the last observation carried forward, or with imputed values following worsening of disease or death, in accordance with the protocol.
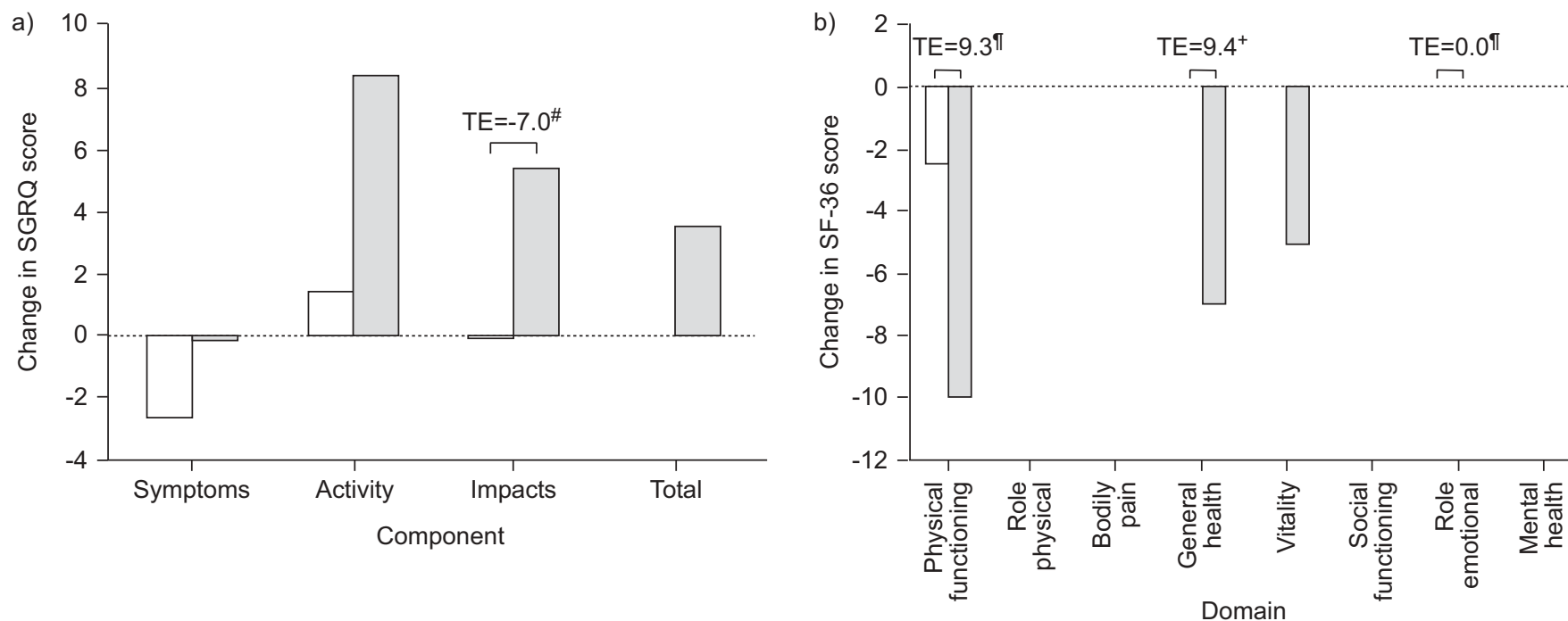

FIGURE 3. Changes from baseline in the surgical lung biopsy subset up to month 12 in: a) St George's Respiratory Questionnaire (SGRQ) component and total scores; and b) 36-item short-form health survey (SF-36) domain scores ( $\square$ : bosentan; $1:$ : placebo). Lower scores indicate improved health-related quality of life (HRQoL) in the SGRQ and higher scores improved HRQoL in the SF-36. Data are presented as medians ( $n=48$ (but, in the placebo group, $n=49$ for symptoms component of $S G R Q$ and $n=47$ for vitality and mental health domains of SF-36)). Treatment effects (TEs) are shown where $p<0.05$, and were estimated using the two-sample Hodges-Lehmann estimator, which estimates the median of the treatment differences. ${ }^{\#}: p=0.03 ;{ }^{\top}: p=0.04 ;{ }^{+}: p=0.01$. 


\begin{tabular}{|c|c|c|c|c|c|}
\hline \multirow[t]{3}{*}{ TABLE 2} & \multicolumn{5}{|c|}{$\begin{array}{l}\text { Number of subjects for whom analyses were performed with the last observation carried forward or with imputed values } \\
\text { in the all-treated population }\end{array}$} \\
\hline & & \multicolumn{2}{|c|}{ Bosentan } & \multicolumn{2}{|c|}{ Placebo } \\
\hline & & M6 & Up to M12 & M6 & Up to M12 \\
\hline Subjects n & & \multicolumn{2}{|c|}{71} & \multicolumn{2}{|c|}{83} \\
\hline SF-36 & & 5 & 4 & 3 & 6 \\
\hline Borg dyspn & ea index & 7 & 9 & 3 & 4 \\
\hline TDI & & 4 & 3 & 1 & 1 \\
\hline \multicolumn{6}{|c|}{ Imputed values } \\
\hline SGRQ & & 4 & 5 & 3 & 6 \\
\hline
\end{tabular}

M: month; SGRQ: St George's Respiratory Questionnaire; SF-36: 36-item short-form health survey; TDI: transition dyspnoea index.

\section{DISCUSSION}

HRQoL and dyspnoea are important components of well-being in patients with IPF. In the present study, data from the BUILD-1 trial were used to assess changes in these two patientreported outcomes over 12 months. Overall, there was relatively little change in either outcome. Among subjects who had undergone SLB for diagnosis of IPF (by identification of the histological pattern of usual interstitial pneumonia), bosentan treatment appeared to have beneficial effects on both HRQoL and dyspnoea.

To date, there have been few large placebo-controlled trials of therapeutic agents for IPF $[6,16,17]$. Neither interferon- $\gamma-1 b$ [17] nor pirfenidone [16] were found to have beneficial effects on HRQoL or dyspnoea. To our knowledge, bosentan is the first agent suggested to show benefit for these important outcomes in patients with IPF.

Often, the absence of honeycombing on high-resolution computed tomography (HRCT) makes a clinical diagnosis of IPF more difficult. Consequently, current guidelines recommend performing SLB in patients with suspected IPF who do not exhibit significant honeycombing on HRCT [8]. One hypothesis generated from the BUILD-1 trial was that the beneficial effects of bosentan would be greater in subjects with less honeycombing on HRCT. In order to investigate this hypothesis, analyses were also performed using data only from subjects whose diagnosis was ascertained by the presence of usual interstitial pneumonia on histological examination of SLB specimens, the SLB subset. Indeed, beneficial effects of bosentan on HRQoL and dyspnoea were suggested by analyses in this subset.

The mechanisms underlying the apparent beneficial effects of bosentan on HRQoL and dyspnoea in the patient subgroup with biopsy-proven IPF observed in the present study are unclear. Treatment with bosentan may delay disease progression and physiological decline, hence influencing HRQoL and dyspnoea. Another consideration is that bosentan may have improved pulmonary vascular parameters, which led to the suggested benefits in patient-centred outcomes. This latter factor seems unlikely since patients with echocardiographic evidence of significant pulmonary hypertension were excluded from the study. We consider the results of the current study to stem from, and mirror, trends observed in a post-hoc analysis of the BUILD-1 trial favouring bosentan [6]. Beneficial trends in physiological parameters and mortality were particularly evident in the subset of subjects with SLB-proven IPF [6]. We hypothesise that these observations arose because these subjects exhibited less extensive fibrosis, made apparent by a lack of significant honeycombing on HRCT.

The present study has a few limitations. The BUILD-1 trial was not powered for evaluation of the HRQoL or dyspnoea outcomes that were used as exploratory end-points; therefore, the sample size may not have been sufficient to detect differences in certain instrument or domain scores. Response data were obtained using the last observation carried forward or imputation during some assessments of HRQoL and dyspnoea in up to 13 subjects. In addition, the between-group differences in change scores were relatively small.

An analysis of subjects who completed the study versus those who discontinued was not performed; however, the proportion of patients who discontinued was similar in both treatment arms. Therefore, substitution rules were applied approximately equally. Although several observations were considered to differ between treatment groups, the CIs in some results covered the published minimum important differences for these instruments. As such, these observations may not carry strong clinical significance. However, the minimum important differences for the SGRQ, SF-36, BDI and TDI have not been established in IPF. It is, therefore, possible that the differences observed here possess clinical importance. Furthermore, the exploratory nature of these analyses should be viewed as hypothesis-generating and should not be considered definitive evidence that bosentan has beneficial effects on these important outcomes. 
Certain domains from the SGRQ and SF-36 exhibited floor and ceiling effects, which may have influenced results in either direction. For example, such effects may have restricted detection of the true underlying improvement in the $33 \%$ of bosentan-treated subjects whose baseline scores in the SF-36 role physical domain were already at 100 , its highest value. Likewise, a floor effect could have occurred if the $36 \%$ of placebo-treated subjects with the lowest baseline score in the role emotional domain declined further, since their scores could not decrease further despite perception of decline. Finally, the responsiveness of the instruments used in the present study is unknown and needs to be determined in future studies.

In conclusion, HRQoL and dyspnoea changed minimally over 12 months. Potential beneficial effects of bosentan on important patient-centred outcomes were identified in the subset of subjects who had undergone SLB as part of their diagnostic evaluation. These observations will be investigated further in the ongoing BUILD-3 trial.

\section{CLINICAL TRIALS}

This study is registered at ClinicalTrials.gov (trial number NCT00071461).

\section{STATEMENT OF INTEREST}

Statements of interest for J. Behr, R.M. du Bois, K.K. Brown, T.E. King Jr, G. Raghu, S. Roux and J. Swigris and the study itself can be found at www.erj.ersjournals.com/misc/statements.dtl

\section{ACKNOWLEDGEMENTS}

The authors would like to gratefully acknowledge all of the investigators involved in the present study: I. Ben-Dov (Sheba Medical Center, Tel Hashomer, Israel), C. Chan (Rosedale PFT Laboratory, Toronto, ON, Canada), J-F. Cordier (Hôpital Louis Pradel, Lyon, France), J. Dauber (University of Pittsburgh, Pittsburgh, PA, USA), J. De Andrade (University of Alabama at Birmingham, Birmingham, AL, USA), A. Frost (Baylor College of Medicine, Houston, TX, USA), T. Geiser (Inselspital, Berne, Switzerland), M. Glassberg (University of Miami/Jackson Memorial Hospital, Miami, FL, USA), J. Golden (University of California San Francisco, San Francisco, CA, USA), G. Hunninghake (University of Iowa Hospitals and Clinics, Iowa City, IA, USA), S. Kalra (Mayo Clinic, Rochester, MN, USA), L. Lancaster (Vanderbilt University Medical Center, Nashville, TN, USA), R. Levy (St. Paul's Hospital, Vancouver, BC, Canada), F. Martinez (University of Michigan Health System, Ann Arbor, MI, USA), K. Meyer (University of Wisconsin-Madison, Madison, WI, USA), J. Mueller-Quernheim (Freiburg University Clinical Centre, Freiburg, Germany), P. Noble (Duke University School of Medicine, Durham, NC, USA), C. Pison (Grenoble University Hospital, Grenoble, France), C. Poirier (Hôpital NotreDame du CHUM, Montreal, QC, Canada), M. Rossman (University of Pennsylvania, Philadelphia, PA, USA), P. Rottoli (Siena University, Siena, Italy), G. Staehler (Löwenstein Clinic, Löwenstein, Germany), D. Valeyre (Hôpital Avicenne, Bobigny, France), A. Wells (Royal Brompton Hospital, London, UK), G. Yung (University of California, San Diego Medical Center, Thornton Hospital, La Jolla, CA, USA), and D. Zisman (University of California, Los Angeles School of Medicine, Los Angeles, CA, USA). The authors are indebted to the coordinators and patients involved with this study. Additionally, the authors acknowledge the editorial assistance of A. Gray (Elements Communications, Westerham, UK), supported by Actelion Pharmaceuticals (Allschwil, Switzerland), during the preparation of this manuscript.

\section{REFERENCES}

1 Bjoraker JA, Ryu JH, Edwin MK, et al. Prognostic significance of histopathologic subsets in idiopathic pulmonary fibrosis. Am J Respir Crit Care Med 1998; 157: 199-203.

2 Nishiyama O, Taniguchi $\mathrm{H}$, Kondoh $\mathrm{Y}$, et al. Health-related quality of life in patients with idiopathic pulmonary fibrosis. What is the main contributing factor? Respir Med 2005; 99: 408-414.

3 Selman M, King TE, Pardo A. Idiopathic pulmonary fibrosis: prevailing and evolving hypotheses about its pathogenesis and implications for therapy. Ann Intern Med 2001; 134: 136-151.

4 Park SH, Saleh D, Giaid A, et al. Increased endothelin-1 in bleomycin-induced pulmonary fibrosis and the effect of an endothelin receptor antagonist. Am J Respir Crit Care Med 1997; 156: 600-608.

5 Shi-Wen X, Denton CP, Dashwood MR, et al. Fibroblast matrix gene expression and connective tissue remodeling: role of endothelin-1. J Invest Dermatol 2001; 116: 417-425.

6 King TE Jr, Behr J, Brown KK, et al. BUILD-1: a randomized, placebo-controlled trial of bosentan in idiopathic pulmonary fibrosis. Am J Respir Crit Care Med 2008; 177: 75-81.

7 Tomioka H, Imanaka K, Hashimoto K, et al. Health-related quality of life in patients with idiopathic pulmonary fibrosis - crosssectional and longitudinal study. Intern Med 2007; 46: 1533-1542.

8 American Thoracic Society, European Respiratory Society. Idiopathic pulmonary fibrosis: diagnosis and treatment. International consensus statement. Am J Respir Crit Care Med 2000; 161: 646-664.

9 Jones P, Quirk F, Baveystock C. The St George's Respiratory Questionnaire. Respir Med 1991; 85: 25-31.

10 Ware JE Jr, Sherbourne CD. The MOS 36-item short-form health survey (SF-36). I. Conceptual framework and item selection. Med Care 1992; 30: 473-483.

11 Schunemann HJ, Griffith L, Jaeschke R, et al. Evaluation of the minimal important difference for the feeling thermometer and the St. George's Respiratory Questionnaire in patients with chronic airflow obstruction. J Clin Epidemiol 2003; 56: 1170-1176.

12 Hays RD, Morales LS. The RAND-36 measure of health-related quality of life. Ann Med 2001; 33: 350-357.

13 Borg GA. Psychophysical bases of perceived exertion. Med Sci Sports Exerc 1982; 14: 377-381.

14 Mahler DA, Weinberg DH, Wells CK, et al. The measurement of dyspnea. Contents, interobserver agreement, and physiologic correlates of two new clinical indexes. Chest 1984; 85: 751-758.

15 Witek TJ Jr, Mahler DA. Minimal important difference of the transition dyspnoea index in a multinational clinical trial. Eur Respir J 2003; 21: 267-272.

16 Azuma A, Nukiwa T, Tsuboi E, et al. Double-blind, placebocontrolled trial of pirfenidone in patients with idiopathic pulmonary fibrosis. Am J Respir Crit Care Med 2005; 171: 1040-1047.

17 Raghu G, Brown KK, Bradford WZ, et al. A placebo-controlled trial of interferon gamma-1b in patients with idiopathic pulmonary fibrosis. N Engl J Med 2004; 350: 125-133. 